\title{
A Novel Solid-acid Catalyst Using Sulfonated Crosslinked Chitosan Resin
}

\author{
C. L. Tang, ${ }^{a}$ L. P. Lv, ${ }^{\text {b,c }}$ Y. T. Xu, ${ }^{a}$ Y. F. Tan,,${ }^{a, k}$ and L. C. Dong, \\ ${ }^{a}$ School of Chemistry and Chemical Engineering, Chongqing University, \\ Chongqing 400044, PR China \\ ${ }^{\mathrm{b}}$ School of Chemistry and Chemical Engineering, Yangtze Normal \\ University, Fuling 408100, PR China \\ 'Research Center for Environmental Monitoring, Hazard Prevention \\ of Three Gorges Reservoir, Yangtze Normal University, Fuling 408100, \\ PR China
}

doi: 10.15255/CABEQ.2015.2279

Original scientific paper Received: July 24, 2015 Accepted: May 26, 2016

Sulfonated crosslinked chitosan resins (SCCRs) were prepared by firstly crosslinking chitosan to crosslinked chitosan resins (CCRs) using a reverse emulsion crosslinking method, followed by sulfonating CCRs with concentrated $\mathrm{H}_{2} \mathrm{SO}_{4}$ as the sulfonation agent. The properties and application of SCCRs as solid acid catalysts were studied. The acidic sites in SCCRs, including $\mathrm{C}_{6}-\mathrm{O}$ and $\mathrm{C}_{2}-\mathrm{N}$ sulfate groups, are all weak acidic sites. SCCRs of higher crosslinking degrees have a higher sulfonation rate of the $\mathrm{C}_{6}$ primary hydroxyl groups, thus more $\mathrm{C}_{6}-\mathrm{O}$ sulfate groups. High-temperature treatment and TG analysis verified that the crosslinking can improve the thermostability of both SCCR backbone and its acidic groups, and a higher crosslinking degree leads to better thermostability. Catalytic esterification of citric acid with butanol and propionic acid with n-butyl alcohol demonstrated that SCCRs have good catalytic activity and can be repetitively used as efficient solid acid catalysts.

Key words:

sulfonated crosslinked chitosan resin, solid acid catalyst, chitosan sulfate, crosslinked chitosan resin

\section{Introduction}

Chitosan is the $N$-deacetylated derivative of chitin, the second most abundant biopolymer next to cellulose in the earth. Besides the advantages of being non-toxic, biocompatible, biodegradable, and highly hydrophilic, chitosan is characterized by a strong affinity to transition metals, which makes it an excellent catalyst-supporting material. Accordingly, a wide range of catalysts supported by chitosan have been developed for uses in the field of hydrogenation, oxidation, and fine chemical synthesis reactions. ${ }^{1,2}$ Recently, considerable attempts have been made to explore the application of chitosan or chitosan derivative itself (without metal immobilization) as catalysts. Valentin et al. ${ }^{3}$ showed that chitosan microspheres, obtained under supercritical $\mathrm{CO}_{2}$ conditions, could be used as a catalyst for the synthesis of monoglyceride from fatty acid and gly-

"Corresponding author: Yunfei Tan, Email: yftan@cqu.edu.cn, Lichun Dong, Email: lcdong72@cqu.edu.cn cidol. Sudheesh et al. ${ }^{4}$ prepared chitosan hydrogel as solid base catalysts for jasminaldehyde synthesis by the condensation of 1-heptanal with benzaldehyde under solvent-free conditions. Reddy et al. ${ }^{5}$ demonstrated that chitosan hydrogel is an efficient organocatalyst for aldol and knoevenagel reactions. Zhao et al. ${ }^{6}$ reported that chitosan covalently bound with quaternary ammonium salts is an efficient and recyclable single-component catalyst for the synthesis of propylene carbonate from propylene oxide and carbon dioxide without any organic solvent or co-catalyst. In all the studies, the chitosan catalyst can be recovered by simple filtration and reused several times without significant loss of activity. Ricci et al. ${ }^{7}$ demonstrated that aerogel microspheres of chitosan is a effective heterogeneous organocatalyst for the asymmetric direct aldol reaction in water.

Acid catalysts are essential for various chemical reactions in industrial hydrocarbon chemistry. Conventional liquid-acid catalysts (such as sulfuric acid) are efficient, but it is costly and difficult to separate them from the homogeneous reaction mixture, thus resulting in abundant nonrecyclable acid 
waste. Solid-acid catalysts can overcome the weakness of liquid-acid catalysts, providing the advantages of low equipment corrosion, ease of product separation, less potential contamination in waste streams, and recyclability of the catalysts. Among a variety of solid-acid catalysts, polymeric resins have been used commercially in many areas including etherification, esterification, transalklation, acylation chemistry due to its high activity at low temperatures. $^{8-11}$ The two main classes of commercial solid-acid resins are styrene-based sulfuric acids (Amberlyst ${ }^{\circledR}$ and Dow type resins) and the perfluorosulfonic acid-based catalysts (Nafion $\left.{ }^{\circledR}\right)$. However, the development of green and sustainable chemistry attracts researchers to explore the utilization of natural biopolymers for eco-friendly solid-acid catalysts; in this regard, chitosan is an ideal candidate due to its multiple advantages. Recently, a couple of investigations reported that chitosan sulfate, prepared by sulfonating chitosan with dilute $\mathrm{H}_{2} \mathrm{SO}_{4}$ in acetic acid, shows good activity in the esterification reactions, demonstrating the promising prospect to prepare chitosan-based solid-acid catalysts. ${ }^{12,13}$

In this paper, crosslinked chitosan resins (CCRs) of different crosslinking degrees were firstly prepared by using an inverse emulsion method and with glutaraldehyde as the crosslinking agent. Then, sulfonated crosslinked chitosan resins (SCCRs) were synthesized by sulfonating CCRs at -5 ${ }^{\circ} \mathrm{C}$ and with concentrated $\mathrm{H}_{2} \mathrm{SO}_{4}$ as the sulfonation agent. Characterizations demonstrated that both the $\mathrm{C}_{2}$ amino and the $\mathrm{C}_{6}$ primary hydroxyl groups in CCRs can participate in the sulfonation reaction, while the SCCR with a higher crosslinking degree has a higher sulfonation rate of the $\mathrm{C}_{6}$ hydroxyl groups. High temperature treatment and thermogravimetric (TG) analysis demonstrated that the crosslinking reaction significantly enhanced the thermostability of SCCRs. Consequently, SCCRs exhibited better catalytic performance and reusability than chitosan sulfate as solid-acid catalysts in catalyzing the esterification of citric acid with butanol and propionic acid with n-butyl alcohol.

\section{Experimental}

\section{Material and reagent}

Chitosan with a deacetylation degree of $90.3 \%$ was purchased from Yuhuan Biochemical Co. Ltd. (Hangzhou, China); Aqueous solution of glutaraldehyde (50 wt $\%$ ), liquid paraffin, Tween- 80 were purchased from Chongqing Chemical Reagent Factory (Chongqing, China); calcium carbonate was purchased from Chongqing Beibei Chemical Reagent Company (Chongqing, China); $\mathrm{HCl}$ (36\%, wt\%),
$\mathrm{H}_{2} \mathrm{SO}_{4}(98 \%$, wt $\%), \mathrm{HNO}_{3}(65 \%$, wt $\%), \mathrm{NaOH}$, chlorosulfonic acid, anhydrous ethanol, acetic acid, butanol, tributyl citrate, n-butyl alcohol, propionic acid, and citric acid were all from Chongqing Chuandong Chemical Reagent Co. Ltd. All the reagents were of Analytical Reagent Grade and used without further purification.

\section{Preparation of crosslinked chitosan resin}

Crosslinked chitosan resin (CCR) was synthesized from chitosan by using the reverse emulsion method reported in literatures with small modification. ${ }^{14-16}$ Firstly, $100 \mathrm{~mL}$ chitosan solution $(2 \%$, $\mathrm{w} / \mathrm{v})$ in $\mathrm{HCl}(0.42 \mathrm{wt} \%)$ was added into the mixtures of $300 \mathrm{~mL}$ paraffin oil and $1.0 \mathrm{~mL}$ Tween- 80 under vigorous stirring using a mechanical stirrer at room temperature. After $30 \mathrm{~min}, 30 \mathrm{~mL}$ glutaraldehyde solution $(5 \%, \mathrm{v} / \mathrm{v})$ and $2.0 \mathrm{~g} \mathrm{CaCO}_{3}$ powder were slowly added to the liquid. After being stirred for $1 \mathrm{~h}$ at $40{ }^{\circ} \mathrm{C}$, the reaction system was adjusted to $\mathrm{pH}=10.0$ using $\mathrm{NaOH}$ solution, and stirred for $3 \mathrm{~h}$ at $60{ }^{\circ} \mathrm{C}$. After vacuum-filtration to separate the liquid and the crude product was washed subsequently with water and ethanol, $20 \mathrm{~mL} \mathrm{HCl}\left(0.2 \mathrm{~mol} \mathrm{~L}^{-1}\right)$ was added to dissolve $\mathrm{CaCO}_{3}$ and form pore network. Finally, the expected CCR product was obtained by washing with D.I. water to remove the excessive $\mathrm{HCl}$, followed by washing thoroughly with petroleum ether and ethanol. For comparison, in this study, CCRs with the theoretical crosslinking degree (proportion of the crosslinked free $\mathrm{C}_{2}$ amino groups calculated by assuming that all the glutaraldehyde had reacted with the free $\mathrm{C}_{2}$ amino groups) of $20 \%, 40 \%, 60 \%, 80 \%$, and $100 \%$ were prepared by controlling the amount of the added glutarldehyde.

\section{Preparation of sulfonated crosslinked chitosan resin}

After being dried in a vacuum oven at $60{ }^{\circ} \mathrm{C}$ for $8 \mathrm{~h}, 4 \mathrm{~g} \mathrm{CCR}$ was added into a three-necked flask, then $30 \mathrm{~mL} \mathrm{H}_{2} \mathrm{SO}_{4}$ solution $(98 \mathrm{wt} \%$ ) was added dropwise at $-5^{2} \mathrm{C}$ under stirring. After the addition was completed, the reaction mixture was continuously stirred for $2 \mathrm{~h}$ at $-5^{\circ} \mathrm{C}$. Subsequently, $200 \mathrm{~mL}$ D.I. water was added into the flask, and the mixture was filtered to obtain the crude SCCR product, which was thoroughly washed with D.I. water, and treated in $100 \mathrm{~mL}$ D.I water at $60{ }^{\circ} \mathrm{C}$ for $4 \mathrm{~h}$. The obtained SCCR product was then dried in a vacuum oven at $60^{\circ} \mathrm{C}$ for $8 \mathrm{~h}$.

\section{Preparation of chitosan sulfate}

Chitosan sulfate (CHS) was prepared by closely following the procedure reported in literature. ${ }^{12,13}$ 
Thus, $2 \mathrm{~g}$ chitosan was dissolved in $100 \mathrm{~mL}$ acetic acid $(2 \%, w / w)$, and then a dilute $\mathrm{H}_{2} \mathrm{SO}_{4}$ solution (20 wt \%) was added under vigorous stirring. After the obtained precipitate was thoroughly washed with D.I. water, ethanol and acetone, the expected product was obtained by drying the precipitate at 60 ${ }^{\circ} \mathrm{C}$ for $8 \mathrm{~h}$

\section{Characterization of catalytic performances}

In this study, the performance of SCCRs and CHS as solid-acid catalysts was explored by studying their activity and reusability to catalyze the esterification of citric acid with n-butanol to produce tributyl citrate from, as well as the synthesis of n-butyl propionate via the esterification of n-butyl alcohol with propionic acid.

For the synthesis of tributyl citrate, the reaction procedure was as follows: $4.2 \mathrm{~g}$ citric acid, $10.5 \mathrm{~mL}$ n-butanol, and SCCR catalysts were slowly added into a three-necked flask equipped with a water separator. The reactions were then processed under reflux and stirring by controlling the temperature with an oil bath. For the synthesis of n-butyl propionate, the reaction procedure was similar, the reactants were slowly added into a three-necked flask equipped with a water separator. The reactions were then processed under reflux and stirring.

By employing a group of single-factor experiments, the optimum operating conditions for the two reactions were obtained (Please see the supplementary information). For the synthesis of tributyl citrate, the optimum conditions determined via experiments were: molar ratio of citric acid and butanol $=1: 5$; mass ratio of SCCR and citric acid $=$ $1: 10$; reaction temperature $=120{ }^{\circ} \mathrm{C}$; reaction time $=8 \mathrm{~h}$. For the synthesis of n-butyl propionate, the optimum conditions were: molar ratio of propionic acid and n-butyl alcohol $=1: 3$; mass ratio of SCCR and propionic acid $=1: 15$; reaction temperature $=$ $120^{\circ} \mathrm{C}$; reaction time $=5 \mathrm{~h}$.

The acid value of the reaction liquid before and after the reaction was measured using a titration method, in order to determine the esterification degree of citric acid according to Eq. 1. GC-MS analysis demonstrates that both reactions have a very high product selectivity ( $\geq 99.3 \%$ ) with very little by-product, therefore, the yield of tributyl citrate (n-butyl propionate) is very close to the esterification degree of citric acid (propionic acid).

\section{Characterization}

Free amino group measurement

The free amino groups in CCR and SCCR were determined by using the formaldehyde- $\mathrm{pH}$ potentiometric titration method. ${ }^{15,17}$ The sample (1 g), pre-heated in a vacuum oven to a constant weight, was put into $100 \mathrm{~mL} \mathrm{HCl}$ solution $\left(0.10 \mathrm{~mol} \mathrm{~L}^{-1}\right)$. Then, the mixture was titrated to $\mathrm{pH}=8.2$ by using a standard $\mathrm{NaOH}$ solution $\left(0.05 \mathrm{~mol} \mathrm{~L}^{-1}\right)$. After 10.0 $\mathrm{mL}$ formaldehyde solution (mass concentration = $36 \%$ ) was added, the mixture was subsequently titrated back to $\mathrm{pH}=8.2$ by using the standard $\mathrm{NaOH}$ solution $\left(0.05 \mathrm{~mol} \mathrm{~L}^{-1}\right)$, the volume of which was recorded as $V_{1}$. Afterwards, a void experiment (no sample was added) was carried out according to the same procedures, and the volume of used $\mathrm{NaOH}$ solution was recorded as $V_{2}$. The content of free amino groups in the sample was then calculated by Eq. 2. The sulfonation rate of the free amino groups can be determined according to the variation of the content of free amino groups before and after the sulfonation.

Amino group content $=0.016 \cdot 0.05 \cdot\left(V_{1}-V_{2}\right) \cdot 100 \%$

\section{BET specific surface area}

Specific surface areas of the SCCR and CHS samples were determined from $\mathrm{N}_{2}$ adsorption isotherms using an ASAP 2020 adsorption analyzer (Micromeritics, USA). Before measurements, the samples were outgassed at $373 \mathrm{~K}$ for $12 \mathrm{~h}$. The surface areas were calculated according to the Brunauer-Emmett-Teller (BET) equation.

\section{Sulfur content and acidic amount}

Sulfur content of the samples was measured by using the $\mathrm{BaSO}_{4}$ method. The basic principle is to totally decompose and dissolve the samples using a mixed acid of $\mathrm{HCl}$ and $\mathrm{HNO}_{3}$ (volume ratio $=3: 1$ ), in which the sulfur was transferred to $\mathrm{SO}_{4}{ }^{2-}$. Then, $\mathrm{BaCl}_{2}$ was added and the produced $\mathrm{BaSO}_{4}$ precipitation was weighed to determine the sulfur content of the samples.

The acidic amount and strength of the samples were determined according to the Chinese National Standard GB8144-87.

Esterification degree $=\left(1-\frac{\text { The acid value of the system after reaction }}{\text { The acid value of the system before reaction }}\right) \cdot 100 \%$ 


\section{Thermostability}

Thermostability of the synthesized SCCR and CHS was characterized by the methods of high temperature treatment and thermogravimetric (TG) analysis.

During the process of high temperature treatment, an 0.5-g sample was put into a flask containing $20 \mathrm{~mL}$ liquid paraffin, then treated by using an oil bath at $80{ }^{\circ} \mathrm{C}, 100{ }^{\circ} \mathrm{C}, 120{ }^{\circ} \mathrm{C}, 140{ }^{\circ} \mathrm{C}$ for $5 \mathrm{~h}$ under stirring. Subsequently, the treated resins were filtrated and thoroughly washed using $95 \%$ ethanol and DI water, and dried at $60{ }^{\circ} \mathrm{C}$ for $5 \mathrm{~h}$, then the mass and sulfur content of the samples were measured to determine the mass retention and sulfur retention.

TG analysis was performed with a TGA/DSC 1 thermal analyzer (Mettler-Toledo, Switzerland) with a heating rate of $2{ }^{\circ} \mathrm{C} \mathrm{min}^{-1}$.

SEM

Morphology of the samples was characterized by using a scanning electron microscope (VEGAII/ LMU, TESCAN).

IR analysis

IR spectrum of the sample was recorded on a MAGMA - IR550 spectrometer (Nicolet Company, USA) using the $\mathrm{KBr}$ pellet method.

\section{Results and discussion}

\section{Synthesis mechanism of SCCR}

The crosslinking of chitosan is a process to form Schiff bonds between the active $\mathrm{C}_{2}$ amino groups of chitosan with glutaraldehyde. ${ }^{18,19}$ By controlling the dose of glutaraldehyde, crosslinked chitosan resins (CCRs) of 5 different theoretical crosslinking degrees $(\mathrm{TCLD}=20 \%, 40 \%, 60 \%, 80 \%$, $100 \%)$ were synthesized, whose real crosslinking degrees (measured proportion of the crosslinked free $\mathrm{C}_{2}$ amino groups) were determined to be 18.5 $\%, 37.6 \%, 58.2 \%, 78.7 \%, 98.7 \%$, respectively, by measuring the free amino groups in CCRs. ${ }^{15,17}$
Among the three functional groups of chitosan, i.e. $\mathrm{C}_{2}$ amino group, $\mathrm{C}_{6}$ primary hydroxyl group, and $\mathrm{C}_{3}$ secondary hydroxyl group, the activity of $\mathrm{C}_{3}$ secondary hydroxyl group is relatively low. ${ }^{20,21} \mathrm{Con}-$ sequently, the sulfonation reaction of CCR mainly occurs at the $\mathrm{C}_{2}$ amino groups and $\mathrm{C}_{6}$ primary hydroxyl groups. The sulfonation of the $\mathrm{C}_{2}$ amino groups by concentrated $\mathrm{H}_{2} \mathrm{SO}_{4}$ solutions occurs in two steps: the amino groups of chitosan are firstly protonated by $\mathrm{H}_{2} \mathrm{SO}_{4}$; then a $\mathrm{SO}_{4}^{2-}$ anion interacts with two $\mathrm{NH}_{3}^{+}$groups to form ionic bridges between them. ${ }^{21,22}$ Therefore, the sulfonation at the $\mathrm{C}_{2}$ amino groups can also realize the crosslinking of chitosan. The sulfonation reaction of the $\mathrm{C}_{6}$ hydroxyl groups is illustrated in Fig. 2b, which is a process in which the hydroxyl groups are esterified to sulfate esters by forming $\mathrm{C}-\mathrm{O}-\mathrm{S}$ bonds.

\section{SCCR and CHS characterization}

\section{IR analysis}

Fig. 3 compares the IR spectra of chitosan, chitosan sulfate, crosslinked chitosan resin (TCLD = $60 \%$ ), sulfonated crosslinked chitosan resin (TCLD $=20 \%, 60 \%, 100 \%)$. In the spectra, the peaks between 2800 and $3000 \mathrm{~cm}^{-1}$ are attributed to the stretching vibration of methyl and methylene groups. The absorption band around $3500 \mathrm{~cm}^{-1}$ is associated with the stretching vibration of the $\mathrm{N}-\mathrm{H}$ groups and the $\mathrm{O}-\mathrm{H}$ groups of the residual moisture. The spectra of chitosan and crosslinked chitosan do not exhibit significant difference since the characteristic peak of the $\mathrm{C}=\mathrm{N}$ bond, which normally appears at $1630-1690 \mathrm{~cm}^{-1}$, overlaps with that of $-\mathrm{NH}_{3}{ }^{+} \cdot{ }^{23}$

Comparing SCCR of high crosslinking degree (TCLD $=60 \%$ and $100 \%$ ) with CCR and chitosan, two strong peaks at $1260 \mathrm{~cm}^{-1}$ and $802 \mathrm{~cm}^{-1}$ appear in the spectrum of SCCR of high TCLD. The peak at $1260 \mathrm{~cm}^{-1}$ is attributed to the $\mathrm{S}=\mathrm{O}$ bond, and that at $802 \mathrm{~cm}^{-1}$ corresponds to the $\mathrm{C}-\mathrm{O}-\mathrm{S}$ bond, indicating the formation of $\mathrm{C}_{6}-\mathrm{O}$ sulfate ester. ${ }^{21-23}$ Moreover, the characteristic adsorptions of the primary alcoholic $\mathrm{C}-\mathrm{O}$ bond and glycoside bond in the saccharide ring are overlapped into a broad peak be-

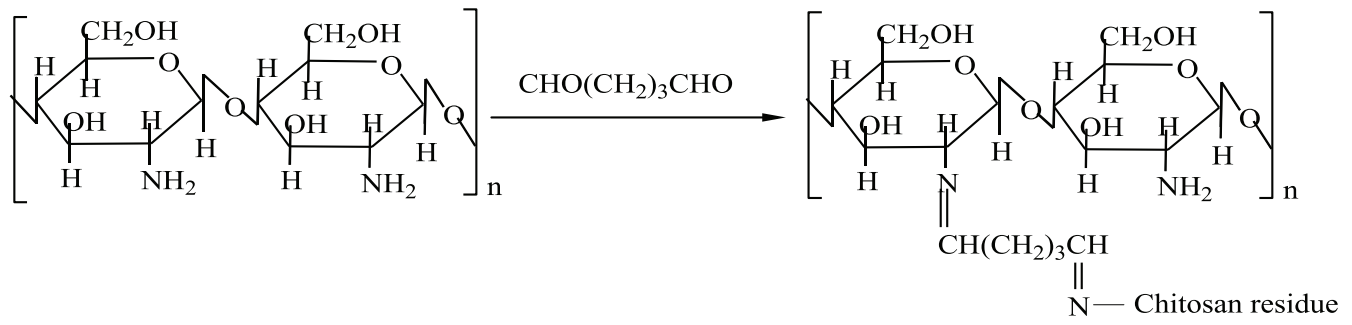




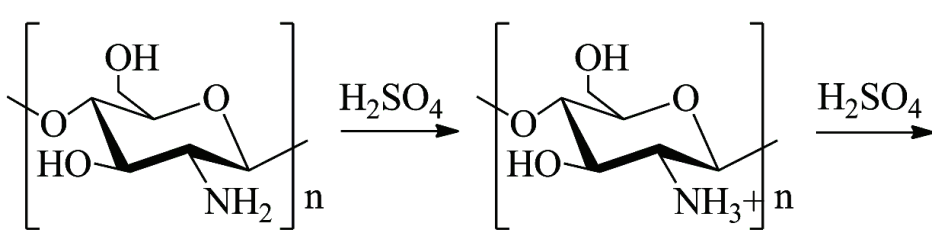

(a)

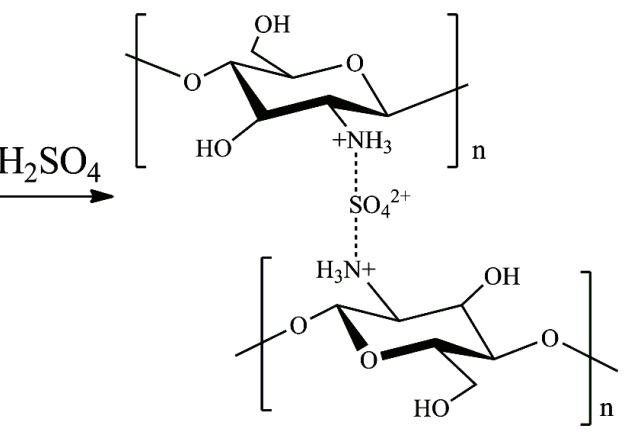
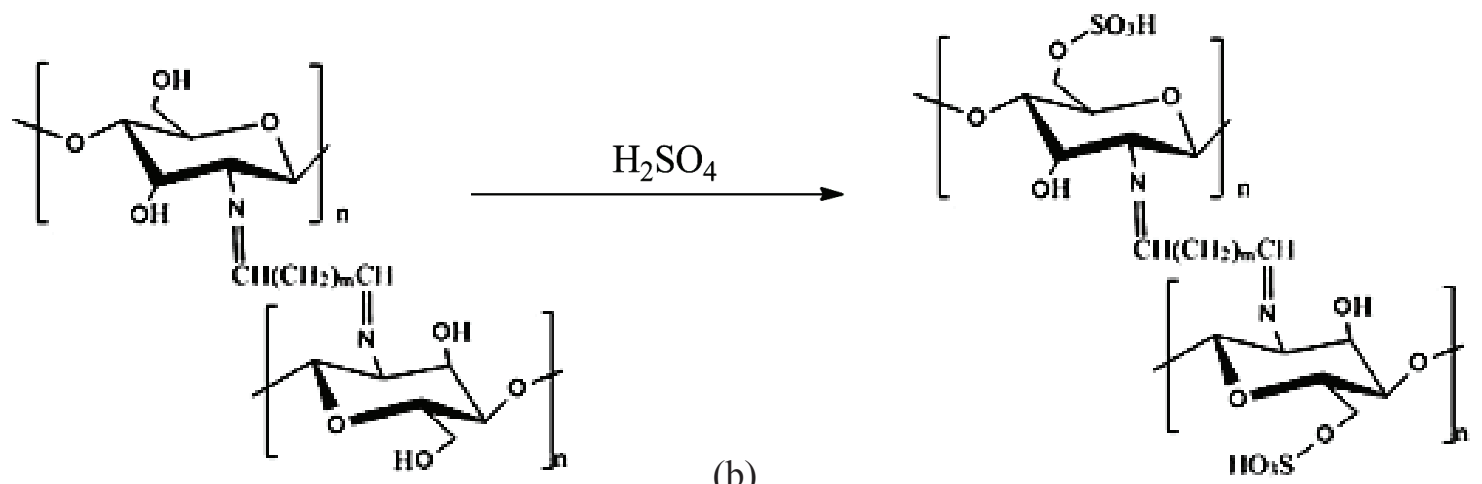

Fig. 2 - Sulfonation reactions of CCR at $C_{2}$ amino groups and $C_{6}$ hydroxyl groups

tween $950 \mathrm{~cm}^{-1}$ and $1100 \mathrm{~cm}^{-1}$ in the spectra of chitosan and CCR, which are split into two peaks in the spectrum of SCCR of high TCLD, the one at $1023 \mathrm{~cm}^{-1}$ is attributed to $\mathrm{C}-\mathrm{O}$ bond, and that at $1096 \mathrm{~cm}^{-1}$ corresponds to glycoside bond. This observation further confirmed formation of $\mathrm{C}_{6}-\mathrm{O}$ sulfate ester in SCCR of high TCLD. ${ }^{24}$

The spectra of CHS and SCCR of TCLD $=20 \%$ exhibit significant difference from that of SCCR of TCLD $=60 \%$ or $100 \%$, in which no obvious adsorption of $\mathrm{C}-\mathrm{O}-\mathrm{S}$ bonds can be found. Moreover, two additional peaks appear at $1500 \mathrm{~cm}^{-1}$ and 660 $\mathrm{cm}^{-1}$. The peak at $1500 \mathrm{~cm}^{-1}$ is attributed to $-\mathrm{NH}_{3}^{+}$, whose adsorption is affected by forming ionic bridges with $\mathrm{SO}_{4}^{2-}$, while the peak at $660 \mathrm{~cm}^{-1}$ probably corresponds to $\mathrm{SO}_{4}^{2-}$. This result indicates that the sulfonation reaction in CHS and CSSR of TCLD $=20 \%$ mainly occurs at the $\mathrm{C}_{2}$ amino groups.

\section{SEM}

Fig. 4 shows the SEM images of SCCR (TCLD $=100 \%$ ) and CHS before and after being reused 5 times as solid-acid catalysts in the synthesis reaction of tributyl citrate from citric acid and butanol. The unused SCCR are near-spherical particles with the diameter around 400-600 $\mu \mathrm{m}$. Since the synthesized SCCR is highly porous before drying, the dried SCCR exhibits multi-fold structures. After being reused 5 times, SCCR can basically keep its structure as the breaking of particles was rarely ob- served. However, it is obvious that the surface of the particles becomes rough due to the surface corrosion and contamination. Unlike the SCCR particles, a significant amount of broken CHS particles was observed after being used 5 times, indicating that SCCR had better mechanical stability than CHS.

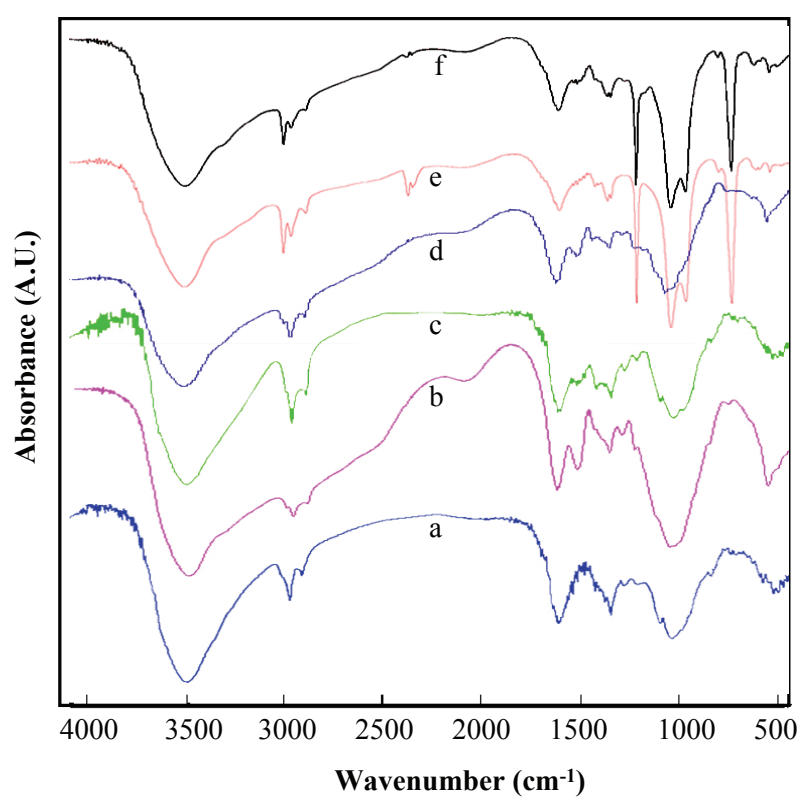

Fig. 3 - IR spectra of CS (a), CHS (b), CCR of TCLD $=60$ $\%(c), S C C R$ of $T C L D=20 \%(d)$, SCCR of TCLD $=60 \%(e)$, $S C C R$ of TCLD $=100 \%(f)$ 


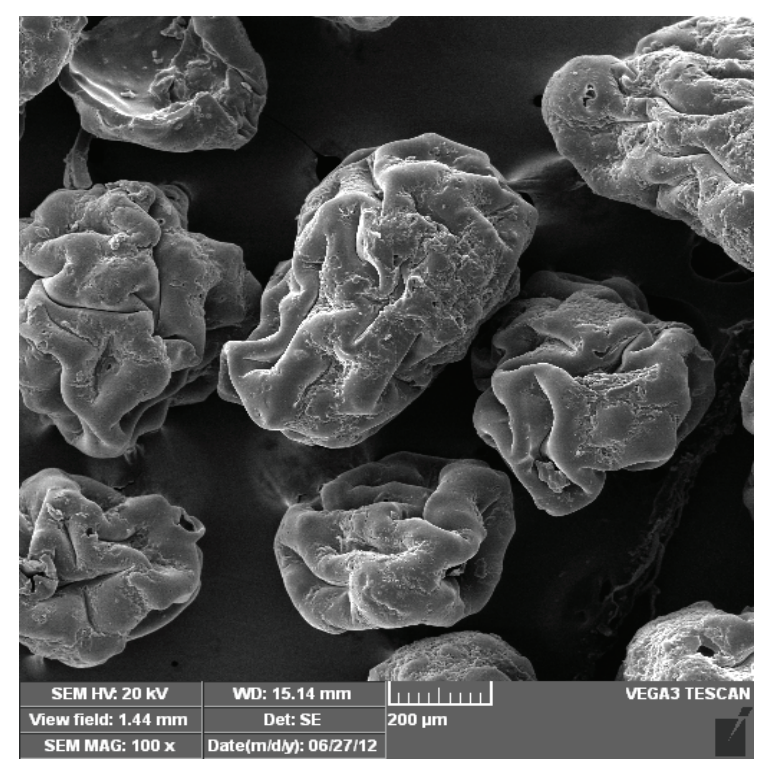

(a)

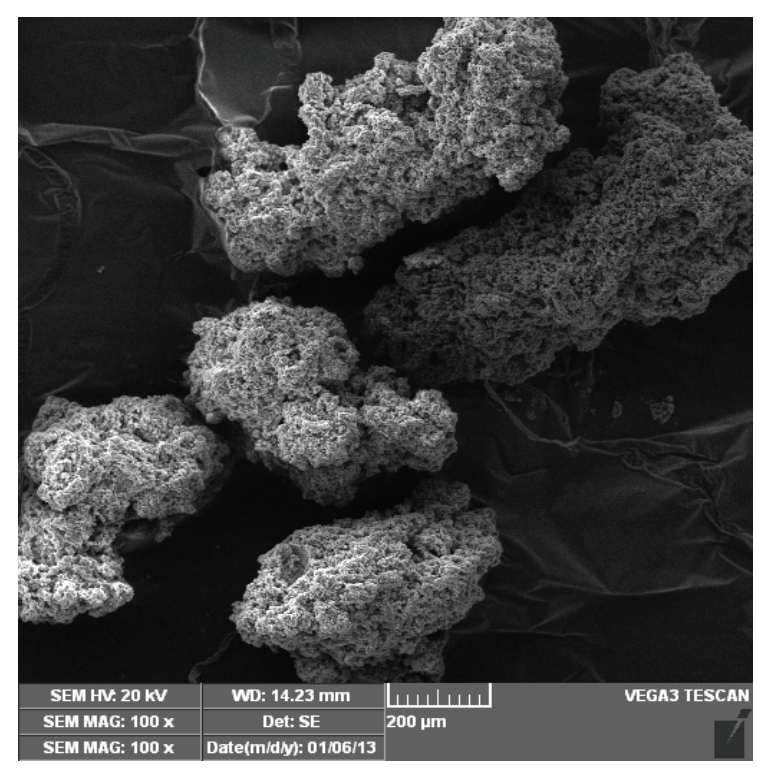

(c)

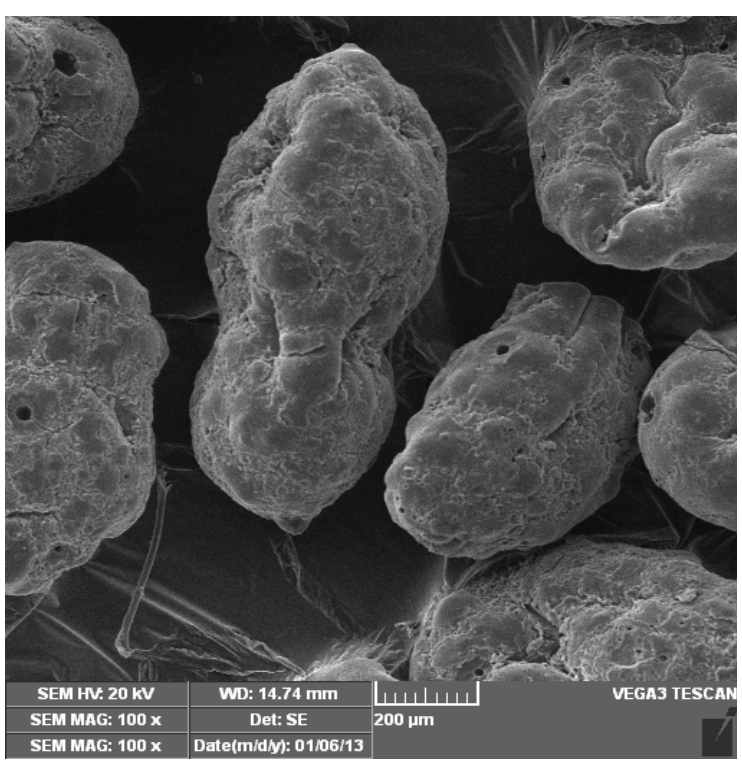

(b)

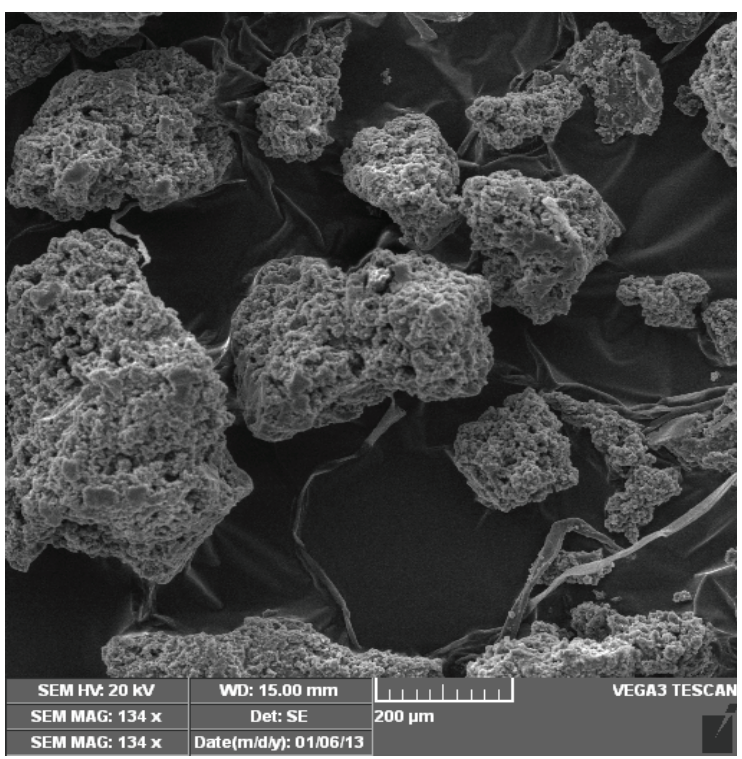

(d)

Fig. 4 - SEM image of SCCR (TCLD = $100 \%)$ and CHS before and after being reused 5 times as solid-acid catalysts in the synthesis reaction of tributyl citrate from citric acid and butanol. (a): SCCR before being used; (b): SCCR after being used; (c): CHS before being used; (d): CHS after being used.

BET specific surface areas

Both the BET specific surface area and average pore size of SCCRs (Table 1) decrease with an increase in the crosslinking degree, which is attributed to the more compact structure at the higher crosslinking degree. Compared with that of SCCRs, the BET specific surface area of CHS is smaller, since the reverse emulsion method employed to prepared CCRs is helpful to increase the specific surface area of the chitosan resin. ${ }^{16,17,25,26}$

Table 1 - BET specific surface areas of SCCRs and CHS

\begin{tabular}{l|c|c|c|c|c|c}
\hline \multicolumn{1}{c|}{ Samples } & CHS & $\begin{array}{c}\text { SCCR } \\
(\mathrm{TCLD}=20 \%)\end{array}$ & $\begin{array}{c}\text { SCCR } \\
(\mathrm{TCLD}=40 \%)\end{array}$ & $\begin{array}{c}\text { SCCR } \\
(\mathrm{TCLD}=60 \%)\end{array}$ & $\begin{array}{c}\text { SCCR } \\
(\mathrm{TCLD}=80 \%)\end{array}$ & $\begin{array}{c}\text { SCCR } \\
(\mathrm{TCLD}=100 \%)\end{array}$ \\
\hline$S_{B E T}\left(\mathrm{~m}^{2} \mathrm{~g}^{-1}\right)$ & 43.1 & 81.4 & 75.5 & 66.7 & 62.9 & 56.7 \\
Average pore size $(\mathrm{nm})$ & 4.1 & 7.6 & 7.1 & 6.3 & 5.7 & 5.3 \\
Pore volume $\left(\mathrm{cm}^{2} \mathrm{~g}^{-1}\right)$ & 0.04 & 0.17 & 0.15 & 0.11 & 0.09 & 0.08 \\
\hline
\end{tabular}




\section{Sulfur and acidic amount}

The sulfonation of chitosan can be executed homogeneously and heterogeneously. In the homogeneous method, chitosan was firstly dissolved in organic solvents before adding the sulfonation agents, e.g. concentrated sulfuric acid, $\mathrm{SO}_{2}, \mathrm{SO}_{3}$, chlorosulfonic acid. While in the heterogeneous method, chitosan was sulfonated directly in solid state. ${ }^{27} \mathrm{CCR}$ is insoluble in organic solvents, accordingly, SCCR was synthesized by sulfonating CCR heterogeneously using concentrated $\mathrm{H}_{2} \mathrm{SO}_{4}$ (98 wt\%) as the sulfonation agent. The sulfonation temperature and time were set to be $-5^{\circ} \mathrm{C}$ and $5 \mathrm{~h}$ based on optimization experiments. Chlorosulfonic acid and the mixed acid of concentrated $\mathrm{H}_{2} \mathrm{SO}_{4}$ and chlorosulfonic acid $(2: 1, \mathrm{v} / \mathrm{v})$ have also been tried as sulfonation agents and demonstrated to induce obvious oxidative degradation of CCR.

Fig. 5 demonstrates that the sulfur content of SCCR decreases with an increase in its crosslinking degree, mainly due to the reduction in its free amino groups. However, it was found that a higher percentage of $\mathrm{C}_{6}$ hydroxyl groups can be sulfonated in the CCR with a higher TCLD by measuring the free amino groups preserved in SCCR (the inset), which agrees with the IR result. Consequently, SCCR with a higher crosslinking degree has a higher $\mathrm{C}_{6}-\mathrm{O}$ sulfonation rate. The reason may be due to the strong hydrophilic property of the free amino groups, resulting in that the $\mathrm{H}_{2} \mathrm{SO}_{4}$ concentration in the microenvironment around CCR molecules is lower than the bulk concentration. A larger number of free amino groups lead to lower $\mathrm{H}_{2} \mathrm{SO}_{4}$ concentration in the microenvironment, consequently, a lower $\mathrm{C}_{6}-\mathrm{O}$ sulfonation rate for SCCR of a lower crosslinking degree.

Since significant degradation of chitosan can be induced, chitosan sulfate cannot be prepared by sulfonating chitosan heterogeneously with concentrated $\mathrm{H}_{2} \mathrm{SO}_{4}$. Accordingly, in this study, CHS was synthesized homogeneously by firstly dissolving chitosan in acetic acid before adding a dilute $\mathrm{H}_{2} \mathrm{SO}_{4}$ solution as the sulfonation agent. ${ }^{23}$ Since the sulfonation of chitosan only occurs at the $\mathrm{C}_{2}$ amino groups as indicated by Fig. 2a, the sulfur content of CHS is much lower than that of SCCR (Fig. 5).

To clarify the application of CHS and SCCR as solid-acid catalysts, the acidic amount and strength of CHS and SCCR were measured according to the Chinese national standard of GB/T 2895-2008. The results in Fig. 6 demonstrate that the acidic groups in SCCR, including $\mathrm{C}_{6}-\mathrm{O}$ and $\mathrm{C}_{2}-\mathrm{N}$ sulfate groups, are all weak acidic sites. Although the acidic amount calculated according to the sulfur content is slightly larger than the measured acidic amount, they show the same trend, i.e. the acidic amount of SCCR de-

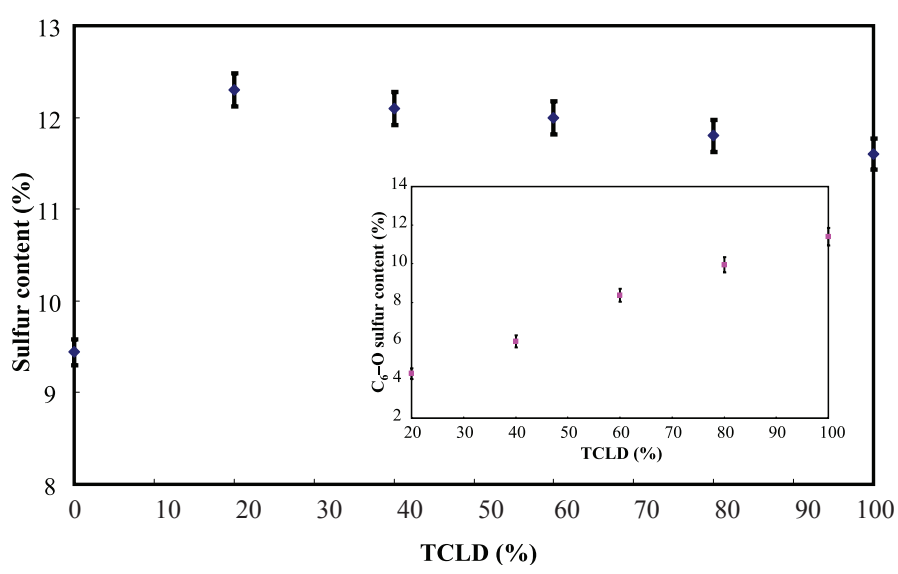

Fig. 5 - Sulfur content (w/w) of SCCR and CHS. The inset shows the sulfur content only accounting for the sulfur in the $\mathrm{C}_{6}-\mathrm{O}$ sulfate ester groups.

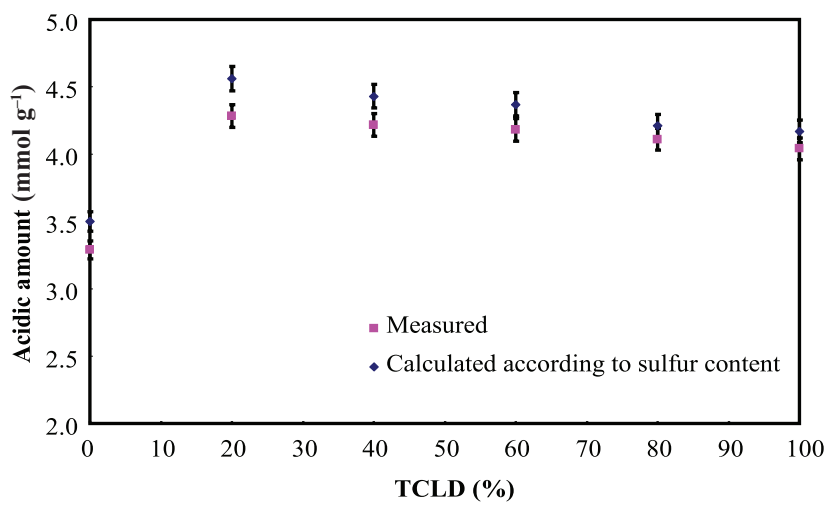

Fig. 6 - Acidic amount of SCCR and CHS

creases with an increase in its crosslinking degree, but all the SCCRs have a larger acidic amount than CHS.

Fig. 8 compares the mass retention of SCCR of different crosslinking degrees after being treated at different temperatures. The result showed that the mass retention of SCCR increases with an increase in its crosslinking degree, demonstrating that SCCR with a higher crosslinking degree has better thermostabiltiy. Moreover, the difference between the thermostability of SCCR of different crosslinking degrees becomes more obvious at the higher temperatures.

With different crosslinking degrees, the thermostability of SCCRs can be varied significantly. TG analysis (Fig. 7) showed that the thermostability of SCCRs increases with an increase in crosslinking degree, indicated by less mass loss of SCCRs with higher crosslinking degrees. Moreover, when the crosslinking degree of SCCRs is higher than $60 \%$, the increase in their thermostability with an increase in crosslinking degrees becomes insignificant. This observation agrees with the result of IR analysis that more $\mathrm{C}_{6}-\mathrm{O}$ sulfate groups had formed in SCCRs with higher crosslinking degrees (> $60 \%$ ). 


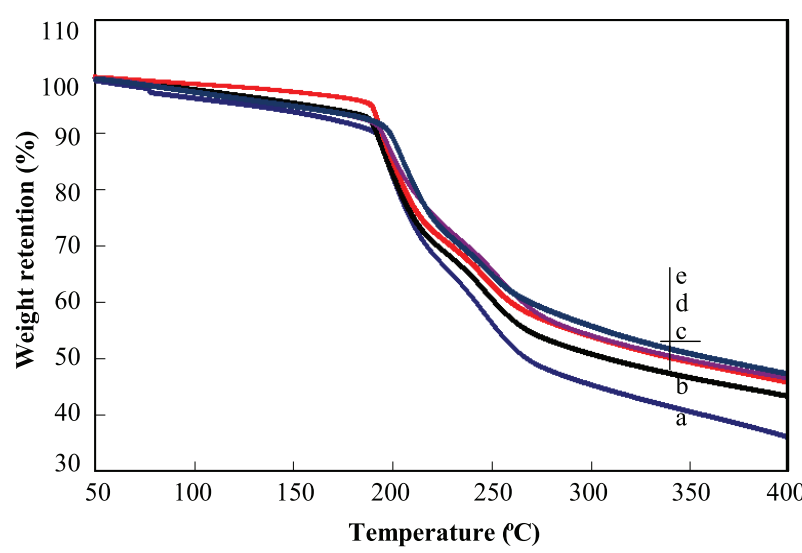

Fig. $7-$ TG curves of SCCR and CHS. $a$ : CHS, $b$ : CSSR with $T C L D=40 \%, c:$ CSSR with TCLD $=60 \%, d: C S S R$ with $T C L D=80 \%$, e: CSSR with TCLD $=100 \%$.

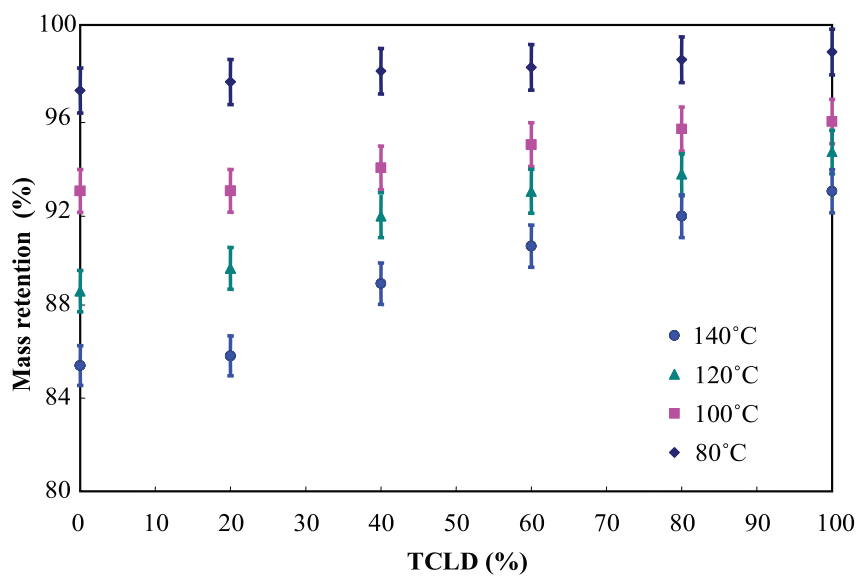

Fig. 8 - Mass retention of SCCR of different crosslinking degrees and CHS after treatment at different temperatures

\section{Thermal stability}

Fig. 9 compares the sulfur retention of unit mass of SCCR treated at the different temperatures, showing that the SCCR of a higher crosslinking degree had larger sulfur retention after treatment. The SCCRs of higher crosslinking degree had a higher sulfonation rate of its $\mathrm{C}_{6}$ hydroxyl groups, thus indicating better thermostability of the $\mathrm{C}_{6}-\mathrm{O}$ sulfate groups than that of $\mathrm{C}_{2}-\mathrm{N}$ sulfate groups.

\section{Catalytic performance of SCCRs and chitosan sulfate}

In this section, the catalytic activity and reusability of SCCRs and CHS as solid acidic catalysts were explored by studying their application in the esterification of citrate with n-butanol to produce tributyl citrate, and the synthesis of n-butyl propionate via the esterification of n-butyl alcohol with propionic acid.

Fig. 10 shows that all the SCCR catalysts exhibit good performance in catalyzing the esterification of citric acid with n-butanol. When the catalysts were used for the first time, the esterification

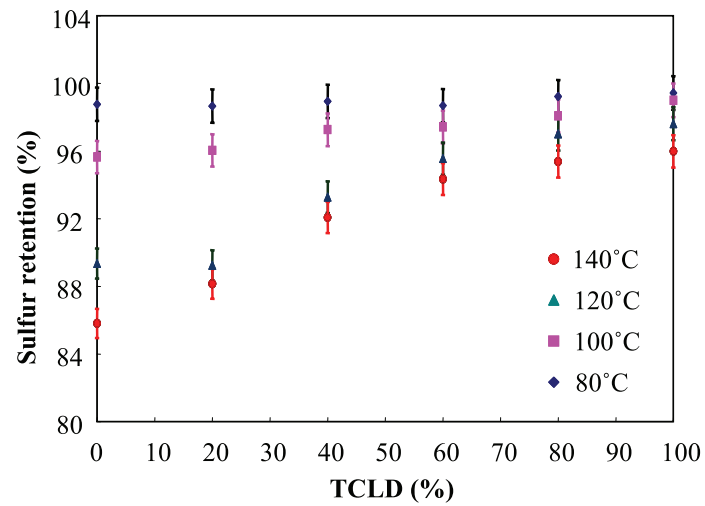

Fig. 9 - Sulfur retention of unit mass of SCCR of different crosslinking degrees and CHS after treatment at different temperatures

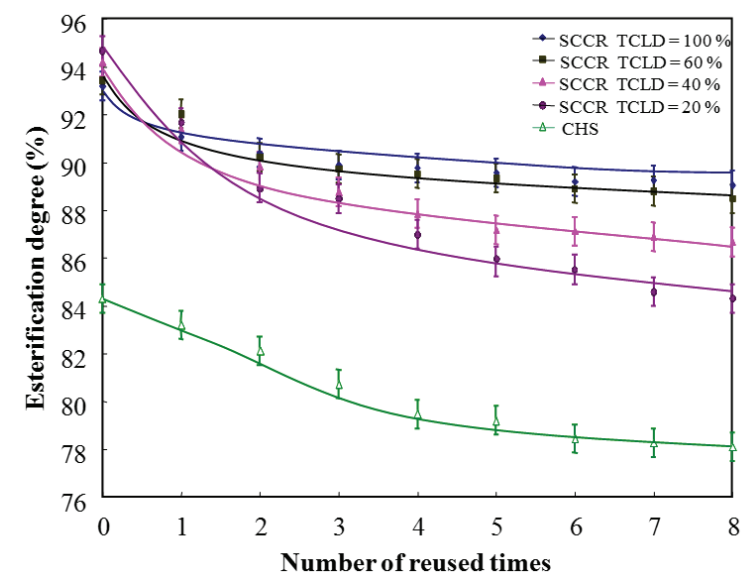

Fig. 10 - Esterification degree of tributyl citrate for the reaction of citric acid with butanol catalyzed by SCCRs and CHS as solid acidic catalysts

degree of citric acid was always higher than $93 \%$, and slightly increased with an increase in the crosslinking degree of SCCR. However, the reusability of the catalysts can be improved by increasing their crosslinking degree. For the SCCR with a theoretical crosslinking degree $=100 \%$, the esterification degree of citric acid decreased only $4.4 \%$ after the catalyst has been reused eight times; whereas for the SCCR with a theoretical crosslinking degree = $20 \%$, the esterification degree of citric acid decreased $11.3 \%$ after the catalyst had been reused eight times. Moreover, the decrease in the catalytic activity of the SCCR with a theoretical crosslinking degree $=100 \%$ mainly occurred during the first three reuses, demonstrating that SCCR with a theoretical crosslinking degree $=100 \%$ had very good reusability as a solid acidic catalyst.

Fig. 10 also compares the catalytic activity of chitosan sulfate as a solid-acid catalyst under the same conditions. The results show that the esterification degree of citric acid reached $84.3 \%$ when CHS was used for the first time; after it was reused 8 times, the esterification degree of citric acid had 


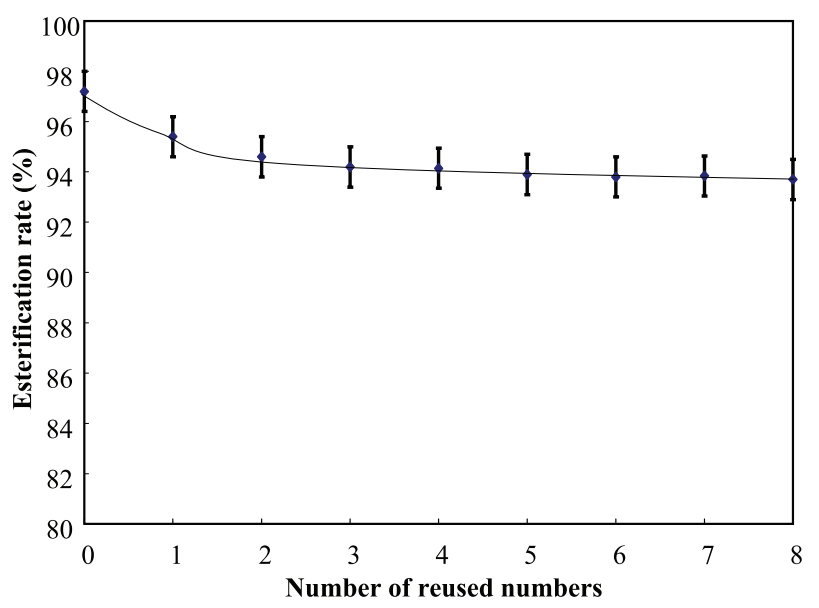

Fig. 11 - Esterification degree of n-butyl propionate for the reaction of $n$-butyl alcohol with propionic acid catalyzed by SCCR (TCLD = $100 \%)$ as solid acidic catalysts

decreased to $78.3 \%$. It is obvious that SCCRs are better solid acidic catalysts than chitosan sulfate.

The result in Fig. 11 shows that the SCCR catalyst $($ TCLD $=100 \%)$ also exhibited good activity and reusability in catalyzing the synthesis of n-butyl propionate via the esterification of n-butyl alcohol with propionic acid. The esterification rate of n-butyl propionate reached $97.1 \%$ when SCCR was used for the first time, and only decreased to $93.6 \%$ after the catalysts were reused 8 times.

The better catalytic activity of SCCR than that of CHS is attributed to its higher acidic amount, while the better reusability is due to its better chemical and thermal stability. As shown in Fig. 6, the acidic amount of SCCR is at least $60 \%$ higher than that of CHS. The glutaraldehyde crosslinking significantly increased the thermal stability of chitosan. ${ }^{13,14}$ Moreover, SCCR contains a larger amount of $\mathrm{C}_{6}-\mathrm{O}$ sulfate groups than $\mathrm{CHS}$, which has better thermostability than $\mathrm{C}_{2}-\mathrm{N}$ sulfate groups.

\section{Conclusions}

Crosslinked chitosan resins (CCRs) with different crosslinking degrees were synthesized by using an inverse emulsion method and with glutaraldehyde as the crosslinking agent, which were further sulfonated at $-5{ }^{\circ} \mathrm{C}$ with concentrated $\mathrm{H}_{2} \mathrm{SO}_{4}$ as the sulfonation agent. The properties and application of the obtained sulfonated crosslinked chitosan resins (SCCRs) as novel solid acidic catalysts were studied and compared with chitosan sulfate, which was prepared by sulfonating chitosan homogeneously in acetic acid with dilute $\mathrm{H}_{2} \mathrm{SO}_{4}$ as the sulfonation agent. IR analysis showed that the sulfonation of CCR can occur at both $\mathrm{C}_{2}$ amino groups and $\mathrm{C}_{6}$ primary hydroxyl groups, while CCRs with higher crosslinking degrees can have more $\mathrm{C}_{6}$ hydroxyl groups to be sulfonated. The sulfur content and acidic amount of SCCRs are significantly increased compared to those of CHS, and the acidic sites in the $\mathrm{SCCR}$, including $\mathrm{C}_{6}-\mathrm{O}$ and $\mathrm{C}_{2}-\mathrm{N}$ sulfate groups, are all weak acidic sites. Moreover, high-temperature treatment and TG analysis verified that the crosslinking has improved the thermostability of both SCCR backbone and its acidic groups, and a higher crosslinking degree leads to better thermostability. The catalytic activity and reusability of the SCCRs and CHS as solid-acid catalysts were explored and compared by studying their application in the synthesis of tributyl citrate from citric acid and butanol, as well as the synthesis of n-butyl propionate via the esterification of n-butyl alcohol with propionic acid. The result showed that SCCRs are efficient solid-acid catalysts with good reusability, the esterification degree of citrate acid can keep above $90 \%$ by repeatedly using the SCCR (theoretical crosslinking degree $=100 \%$ ) for 8 times, while the esterification degree of n-butyl propionate only decreased from $97.3 \%$ to $93.6 \%$ after the catalyst was reused 8 times.

\section{ACKNOWLEDGMENT}

This work was financially supported by the $\mathrm{Na}$ tional Natural Science Foundation of China (21176270 \&51272296).

\section{References}

1. Mourya, V. K., Inamdar, N. N., Chitosan-modifications and applications: Opportunities galore, React. Funct. Polym. 68 (2008) 1013

doi: http://dx.doi.org/10.1016/j.reactfunctpolym.2008.03.002

2. Guibal, E., Heterogeneous catalysis on chitosan-based materials: a review, Prog. Polym. Sci. 30 (2005) 71. doi: http://dx.doi.org/10.1016/j.progpolymsci.2004.12.001

3. Valentin, R., Molvinger, K., Quignard, F., Brunel, D., Supercritical $\mathrm{CO}_{2}$ dried chitosan: an efficient intrinsic heterogeneous catalyst in fine chemistry, New J. Chem. 27 (2003) 1690 . doi: http://dx.doi.org/10.1039/b310109f

4. Sudheesh, N., Sharma, S. K., Shukla, R. S., Chitosan as an eco-friendly solid base catalyst for the solvent-free synthesis of jasminaldehyde, J. Mol. Catal. A: Chem. 321 (2010) 77.

doi: http://dx.doi.org/10.1016/j.molcata.2010.02.005

5. Reddy, K. R., Rajgopal, K., Maheswari, C. U., Kantam, M. L., Chitosan hydrogel: A green and recyclable biopolymer catalyst for aldol and Knoevenagel reactions, New J. Chem. 30 (2006) 1549. doi: http://dx.doi.org/10.1039/b610355c

6. Zhao, Y., Tian, J., Qi, X., Han, Z., Zhuang, Y., He, L., Quaternary ammonium salt-functionalized chitosan: An easily recyclable catalyst for efficient synthesis of cyclic carbonates from epoxides and carbon dioxide, J. Mol. Catal. A: Chem. 271 (2007) 284.

doi: http://dx.doi.org/10.1016/j.molcata.2007.03.047 
7. Ricci, A., Bernardi, L., Gioia, C., Vierucci, S., Robitzer, M. Quignard, F., Chitosan aerogel: a recyclable, heterogeneous organocatalyst for the asymmetric direct aldol reaction in water, Chem. Commun. 46 (2010) 6288. doi: http://dx.doi.org/10.1039/c0cc01502d

8. Harmer, M. A., Sun, $Q$., Solid acid catalysis using ion-exchange resins, Appl. Catal. A-Gen. 221 (2001) 45. doi: http://dx.doi.org/10.1016/S0926-860X(01)00794-3

9. Climent, M. J., Corma, A., Iborra, S., Heterogeneous Catalysts for the One-Pot Synthesis of Chemicals and Fine Chemicals, Chem. Rev. 111 (2011) 1072. doi: http://dx.doi.org/10.1021/cr1002084

10. Granados, M. L., Alba-Rubio, A. C., Sadaba, I., Mariscal, R., Heras, A., Poly(styrenesulphonic) acid: an active and reusable acid catalyst soluble in polar solvents, Green Chem. 13 (2011) 3203. doi: http://dx.doi.org/10.1039/c1gc15461c

11. Gelbard, G., Organic synthesis by catalysis with ion-exchange resins, Ind. Eng. Chem. Res. 44 (2005) 8468 doi: http://dx.doi.org/10.1021/ie0580405

12. Zhang, X., Yu, Y., Liu, D., Liu, C., Luo, G., Green synthesis of n-butyl propionate catalyzed by chitosan sulfate, Flavour Fragrance Cosmetics 2 (2007) 14. (in Chinese)

13. Wang, K., Xun, J., Wen, H., Synthesis of n-Butyl acetate on chitosan sulfate catalyst by microwave irradiation, Petrochemical Technology, 34 (2005) 364. (in Chinese)

14. $Y u, Y$. $H e, B$., A new type of ALSS - the preparation of crosslinked chitosan resins and its adsorption properties for bilirubin, React. Funct. Polym. 31 (1996) 195. doi: http://dx.doi.org/10.1016/1381-5148(96)00051-X

15. Xiao, Y., Zhou, X., Synthesis and properties of a novel crosslinked chitosan resin modified by L-lysine, React. Funct. Polym. 68 (2008) 1281. doi: http://dx.doi.org/10.1016/j.reactfunctpolym.2008.06.015

16. Dong, L., Wang, G., Xu, Y., Zhou, X., Xiao, Y., Jiang, H., Luo, $Q$., Immobilization of glucose oxidase on a novel crosslinked chitosan support grafted with L-lysine spacers, Chem. Biochem. Eng. Q. 25 (2011) 395

17. Ning, Z., Analysis manual for food ingredients, China Light Industry Press, Beijing, 1998, pp 119-120.

18. Xiang, Y., Yang, M., Guo, Z., Cui, Z., Alternatively chitosan sulfate blending membrane as methanol-blocking polymer electrolyte membrane for direct methanol fuel cell, J. Memb. Sci. 337 (2009) 318.

doi: http://dx.doi.org/10.1016/j.memsci.2009.04.006

19. Wan Ngah, W. S., Fatinathan, S., Chitosan flakes and chitosan - GLA beads for adsorption of p-nitrophenol in aqueous solution, Colloids Surf. A. 277 (2006) 214. doi: http://dx.doi.org/10.1016/j.colsurfa.2005.11.093

20. Rinaudo, M., Chitin and chitosan: Properties and applications, Pro. Polym. Sci. 31 (2006) 603. doi: http://dx.doi.org/10.1016/j.progpolymsci.2006.06.001

21. Jayakumar, R., New, N., Tokura, S., Tamura, H., Sulfated chitin and chitosan as novel biomaterials, Int. J. Biol. Macromol. 40 (2007) 175. doi: http://dx.doi.org/10.1016/j.ijbiomac.2006.06.021

22. Zheng, C., Yan, X., Jiangju, S., Meng, Y., Qi, Z., Tao, Z., Ionic interactions between sulfuric acid and chitosan membranes, Carbohydr. Polym. 73 (2008) 111. doi: http://dx.doi.org/10.1016/j.carbpol.2007.11.009

23. Ellis, G., Marco, C., Gómez, M., Highly resolved transmission infrared microscopy in polymer science, Infrared Phys. Technol. 45 (2004) 349. doi: http://dx.doi.org/10.1016/j.infrared.2004.01.015

24. Zhou, H., Qian, J., Wang, J., Yao, W., Liu, C., Chen, J., $\mathrm{Cao}, \mathrm{X}$., Enhanced bioactivity of bone morphogenetic protein-2 with low dose of 2-N, 6-O sulfated chitosan in vitro and in vivo, Biomaterials 30 (2009) 1715. doi: http://dx.doi.org/10.1016/j.biomaterials.2008.12.016

25. Karine, M., Françoise, Q., Daniel, B., Michel, B., Jean-Marie, $D$., Porous chitosan-silica hybrid microspheres as a potential catalyst, Chem. Mater. 16 (2004) 3367. doi: http://dx.doi.org/10.1021/cm0353299

26. Fabrice, B., Laurent, V., Laurent, D., Alain, D., Thierry, D., A novel synthesis of chitosan nanoparticles in reverse emulsion, Langmuir 24 (2008) 11370. doi: http://dx.doi.org/10.1021/la801917a

27. Zhang, K., Helma, J., Peschel, D., Gruner, M., Groth, T., Fischer, S., NMR and FT Raman characterisation of regioselectively sulfated chitosan regarding the distribution of sulfate groups and the degree of substitution, Polymer. 51 (2010) 4698. doi: http://dx.doi.org/10.1016/j.polymer.2010.08.034 